\title{
White Spaces Communications in Europe
}

\author{
António Morgado and Nuno Borges Carvalho \\ Departamento de Electrónica, Telecomunicações e Informática, \\ Instituto de Telecomunicações da \\ Universidade de Aveiro, \\ Aveiro-Portugal \\ morgado@ua.pt and nbcarvalho@ua.pt
}

\begin{abstract}
Cognitive Radio Communications and the switch off of analog TV in Europe is creating a strong and viable solution for testing new approaches to the implementation of Cognitive Radios in an effective way.

In this paper the concept of White Spaces and specially the approaches that are thought to be developed in Europe will be studied and analyzed. It is expected that this work will be the basis for more focus on the white space communication protocols.
\end{abstract}

\section{Introduction}

Wireless communications continue to populate everywhere, from the local area computer networks, to cellular and broadcasting segments, including wireless sensors and RFID. One common fundamental resource in this technology is spectrum.

Currently we observe a spectrum paradox: many wireless technologies are experiencing spectrum congestion, yet measurement campaigns demonstrate spectrum is being underutilized. In fact, in some regions, at some time of the day, a large amount of frequencies are not being utilized at all. This gives rise to the so called spectrum 'white spaces'. It would be economic and socially advantageous to change the current regulation policy of allocating spectrum to a technology and company, to a more flexible scheme which makes efficient use of these white spaces. One solution is to allow spectrum trading; the other is to encourage the use of 'white spaces' by unlicensed devices.

Moreover the TV broadcasting technology is facing a moment of change, with analogue TV transmissions being gradually shut down and replaced by their digital counterparts almost all over the world. This 'digital switch over' is going to be finished in the end of 2012 in Europe. The need for coexistence of analogue and digital TV during the transition, the different requirements imposed by digital terrestrial TV (DTT) and its better spectral efficiency, demand regulators intervention. This opens a window of opportunity for future spectrum regulation policy change. Regulators are working to provide new rules which allow unlicensed use of 'white spaces' while assuring licensed spectrum owners will not be hassled. In Europe this is quite important and decisive, since the pressure from mobile operators is tailoring the future of these spectra.

\section{Digital TV Switch Over}

Since the early 1920's TV development has occurred in parallel in USA, Europe and Japan [1].

In Japan and Europe the main focus until middle 1990's was on improving analogue systems until reaching high-definition TV (HDTV) systems.

In USA, the same thing was happening, but in $1990 \mathrm{FCC}$ asked for a HDTV system using the same $6 \mathrm{MHz}$ channel used by standard definition analogue TV. This challenge was met through compression techniques and migration to digital technology. Japan and Europe were also working on digital TV, but until middle 1990's only for satellite and cable segments [1].

Digital Terrestrial TV (DTT) systems are being proposed since early 90's, with the first technical standards approved in 1995 (USA). In Europe and Japan the standardization produced the first norm in the late 90's, and the first emissions began in 1998 and 2003 respectively. Since then analogue coexists with digital, but is smoothly being replaced almost all over the world. Some countries have already completely switched-off analogue transmissions, as Luxembourg (2006), Netherlands (2006), Finland (2007), Sweden (2007), Germany (2008), USA (2009). Other countries are expected to follow, like Canada (2011), Japan (2011), most European Countries (2012), South Korea (2012), Australia (2013), China (2015) and Brazil (2016). 
This digital switch over will impose huge amount of free spectra, which could be used efficiently by cognitive radio transceivers, and thus optimize the use of spectra.

\section{Digital TV White Spaces Regulation in Europe}

Digital switch over will free considerable amounts of spectrum in VHF and UHF bands. A smaller amount of this spectrum should be allocated to the more spectral efficient digital TV. The free TV channels, some MHz wide, would be adequate for both supplanting spectrum congestion and allow the florescence of new broadband services. Actually $3^{\text {rd }}$ generation mobile communications operate in $5 \mathrm{MHz}$ channels and WiFi uses $20 \mathrm{MHz}$ bandwidth channels. The VHF and UHF bands are also attractive because of their better propagation characteristics (e.g. range about 3 times higher than in ISM band) and better propagation through walls.

Regulators must decide if they should license the white spaces or allowing their unlicensed use. Licensed use would make difficult the licensing process (auction, beauty contest) and the posterior license supervision. Moreover, the necessity to avoid interference on broadcasting systems imposes additional technological difficulties and costs that could prevent interest in a licensed basis. Unlicensed use of white spaces seems to be preferable as it will make white spaces exploitation cheaper, allowing a greater number of companies to propose innovative uses of the spectrum and a faster adoption rate [2]. It seems there is a place for innovation, which can provide economic benefits for those interested to apply.

Instructed by the respective governments, regulators started to work on regulations on the use of TV white spaces several years ago. Among them, it seems proposals from the United States of America and the European Union are those which are already finished or close to being finished.

Following a 2007 European Commission (EC) mandate, in 2008 the Electronic Communications Committee (ECC), under the European Conference of Postal and Telecommunications Administrations (CEPT), issued a report [3] acknowledging the need for further studies on $470-862 \mathrm{MHz}$ band white space use by Cognitive Radio (CR) devices before deciding to proceed to a European recommendation on that matter.

According to [3] white spaces devices (WSD) should not be protected form interference among them. However, they must not interfere with licensed primary users, and move to other white spaces channels whenever necessary. Among services to protect in $470-862 \mathrm{MHz}$ band are digital television broadcasting (according to Genève 2006 Plan, there will be 7 or 8 multiplexes in most European countries), aeronautical radio navigation, military applications (channel 36), radio astronomy (channel 38), program making and special events services - PMSE (channel 69). Meanwhile, sub-band $790-862 \mathrm{MHz}$ was reserved for mobile communications and the European white spaces band became 470-790 MHz.

Potential candidates for white space use are PMSE devices, other portable devices with output powers up to $100 \mathrm{~mW}$ (WiFi cards, smart phones, media players), and fixed devices with output powers up to $1 \mathrm{~W}$ (broadband wireless access points). Due to the high heterogeneity of scenarios possible when using white spaces, a new technology - cognitive radio - seems specially suited to make useful use of this spectrum [3].

At the $22^{\text {nd }}$ ECC Meeting, held on March 2009, it was requested to form working group SE43 under WG-SE. This group should "define technical and operational requirements for the operation of cognitive radio systems in the white spaces of the UHF broadcasting band (470-790 MHz) to ensure the protection of incumbent radio services/systems and investigate the consequential amount of spectrum potentially available as 'white space, ", [4]

Studies conducted by SE43 considered three WSD groups, all of them using OFDM modulation: personal/portable devices, home/office devices and private/public access points [5] .

The first two groups represent low power, small sized devices with low antenna height. The distinction between them is that portable devices can be moving, while home/office devices are static. Private/public access points are static devices, Internet connected, eventually operating with high power and high antenna heights. All of them can have additional transceivers capable of communicating using bands outside 470-790 MHz.

These three types of devices can operate in several scenarios concerning indoor/outdoor communication, fixed/moving radios and high/low antennas. SE43 considered scenarios are:

Infrastructure like scenarios (i.e. at least, one of the communicating devices is a static access point):

- Outdoor, one access point, both antennas with low height;

- Indoor, one access point, both antennas with low height;

- Outdoor, one access point, one high antenna and one low antenna;

- Outdoor, two access points, both antennas with high height.

Ad-hoc scenarios (i.e. none device is a static access point)

- Outdoor, both antennas with low height. 
Although not mandatory, in the studies conducted in SE43, OFDM modulation was considered for the WSD, as this is the most flexible, efficient and reliable modulation at the moment. Duplex mode can be TDD or FDD, and channel aggregation should be possible to increase throughput. Transmit powers in the order of $10 \mathrm{~mW}$ to $50 \mathrm{~mW}$ are envisioned for short range communications while powers between $1 \mathrm{~W}$ and $10 \mathrm{~W}$ could be allowed for long range transmissions [5]. These signal characteristics should be adapted according to the licensed users that must be protected in a given region.

WSD devices may operate in a collaborative or non collaborative approach to get information about the most appropriate channel to use. In a non collaborative strategy, information on the white space channels can be obtained through spectrum sensing, geo-location plus database enquiry, or accessing local beacon information.

In that sense let us try to understand the way white space spectrum occupancy can be developed.

\subsection{Spectrum sensing}

Spectrum sensing consists in scanning several adjacent channels in order to decide if some of them are or are not being used by licensed users. Although the most practical solution, spectrum sensing suffers from the hiddenterminal problem, which makes necessary to detect extremely low signal levels. Detection algorithms are classified as energy detection or signal feature detection (e.g. pilot carrier/symbols, frequency/time periodicity...). Therefore, many algorithms rely in the intrinsic characteristics of the signal to detect, which means different algorithms and sensing levels should be devised for each technology to protect. Some technologies, such as radio astronomy are not suitable for detection. Even when detection is possible, the existence of protected licensed users in channels near the ones being scanned substantially degrades the performance of sensing algorithms. This may be caused by the fact that the licensed users out-of-band emissions are making the detectors believe the channel is occupied.

In conclusion, with technology available today, spectrum sensing is not yet reliable enough. Maybe the emergence of cooperative sensing brings decisive spectrum sensing improvements.

Even if the sensing devices were able to correctly identify the available channels, they still have to calculate their in-band and out-of-band emission power in order to avoid excessive co-channel and adjacent channel interference on protected users.

- In-band WSD power: a first in-band power value should be computed to assure adequate protection to a DTT user, considering it has the lowest signal level possible, detected in the co-channel or adjacent channels. Following, a second value should be computed, this time to avoid DTT receiver overloading. The in-band WSD power should be set to the lower of these two values [5].

- Out-of-band WSD power: it can be computed taking into account the in-band WSD power and DTT receivers' selectivity, in order to protect the eventual DTT signals in adjacent channels.

However, autonomous WSD can not calculate these power values as it has no knowledge of some information needed to perform the calculations. Specifically, it does not know some propagation parameters such as distance between WSD and DTT emitter, terrain shape and clutter along the path and DTT antenna radiation pattern. One approach to solve this consists in using conservative values for the unknown parameters. But this would result in extremely low WSD transmission power which would make their use not useful. Once again, cooperation between WSD could allow estimating the lacking information in order to compute higher WSD emitter powers with high levels of confidence.

A possible alternative to this analysis is to investigate technologies which allow characterizing the physical distance to obstacles in the vicinity of WSD (e.g.: buildings, trees or people agglomeration, and using that info to get appropriate correction factors to emission power from a lookup table - investigate measurement (distance, volume) devices using an useful technique.

Currently, WSD interference parameters on adjacent channels (ACLR) are also not known as these depend on the modulation used. If OFDM modulation is assumed, some authors [5] are proposing to extrapolate ACLR values from those of LTE signals. Nevertheless, given these difficulties, maximum transmission power is not being set for WSD sensing only devices.

Actually what is being considered at the moment for spectrum sensing is that [5]:

"For the preceding reasons it can be concluded that the Sensing technique investigated, employed by a stand-alone WSD (autonomous operation), does not appear to be reliable enough to guarantee" neither "a correct identification of available channels at a given location " nor to calculate the appropriate emission power "to avoid causing interference to possible nearby co-channel coverage areas. The use of a geo-location database appears, therefore, necessary." 


\subsection{Geo-location plus database}

Other solution for white space use is the geo-location plus database solution, in this case the white space device communicates its position to the database, using other media than the white space channel. The database would respond with the white space channels available and the maximum e.i.r.p. allowed in each channel. Therefore it will be possible to efficiently protect licensed users as long as the appropriate set of parameters and algorithms are used to compute the required information. In [5] some suggestions are made, although each country should determine the parameters and algorithms that are best suited to their national environment. Some other implementation issues remain open, like the data the WSD should provide to the database and how often.

Obviously, geo-location plus database scheme faces additional implementation difficulties, as it needs the existence of geo-location capabilities and reliable internet connection in the devices, and also needs information on protected users to build a database. However these challenges can be alleviated by operating the several devices in a master-slave architecture, where the master accesses the database and then instructs the simpler slave devices on what channels to transmit.

In the end, geo-location can achieve a very low probability of causing interference into protected users.

The database should store, or be able to compute:

- DTT reception signal level in each region (accuracy of 100x100m suggested) with a given confidence level (e.g. signal level above $-68 \mathrm{dBm}$ with $95 \%$ probability)

- Store metrics for decision to allow or not allow using a free channel:

- It is proposed [5] to specify the maximum confidence level reduction on DTT coverage signal level imposed by WSD transmission (e.g. signal level above $-68 \mathrm{dBm}$ with $70 \%$ probability), in our opinion this is not a feasible solution.

- We actually propose that a maximum level of interference is allowed in co-channel and adjacent channel, one possibility could be the use of a set of lookup tables for each protected technology and WSD modulation. There should be tables for protected users in 3 reference channels, with 3 reference power levels, and should be provided maximum interference power levels in the edges of the adjacent channels. Open question continues to be the spurious emissions when there are pair of interferes in adjacent channels and overloading DTT transmitters.

- Algorithms to calculate the WSD power which would produce an interference level below the maximum allowed levels, in the several channels, for the given WSD modulation and protected technology, given the geometry of the situation: distances, environments.

Other solutions for white spaces include for instance beacon transmission, and that will also be explained and discussed in the final paper.

\section{References}

1. Yiyan Wu, Shuji Hirakawa, Ulrich Reimers, Jerry Whitaker, "Overview of Digital Television Development Worldwide”, Invited Paper, Proceedings of the IEEE, Vol. 94, No. 1, January 2006, pp 8-21.

2. Richard Thanki, "The economic value generated by current and future allocations of unlicensed spectrum - Final report", Perspective Associates report for Microsoft, September 2009.

3. Electronic Communications Committee (ECC), "Technical considerations regarding harmonisation options for the Digital Dividend”, CEPT Report 24, June 2008.

4. SE43 Chairman, "Terms of Reference and Work Item for SE43”, Doc. SE43(09)01, May 2009.

5. Electronic Communications Committee (ECC), "Technical and operational requirements for the possible operation of cognitive radio systems in the 'white spaces' of the frequency band 470-790 MHz", ECC Report 159, January 2011. 